\title{
Constrained Robot Control Using Control Barrier Functions
}

\author{
Manuel Rauscher, Melanie Kimmel and Sandra Hirche
}

\begin{abstract}
Many robotic applications, especially if humans are involved, require the robot to adhere to certain joint, workspace, velocity or force limits while simultaneously executing a task. In this paper, we introduce a control structure, which merges an arbitrary desired robot behavior with given constraints. Using a quadratic program (QP), control barrier functions (CBFs) are combined with an arbitrary nominal control law, which determines the desired behavior. The CBFs enforce the constraints, overruling nominal control whenever necessary. We show that the concept is applicable with arbitrary numbers of constraints and any nominal control law. In order to illustrate the capabilities of the approach, the control scheme is applied to an anthropomorphic manipulator, which is constrained by static as well as moving constraints.
\end{abstract}

\section{INTRODUCTION}

While nowadays robotic systems are commonly found in industrial production lines where their workspaces are separated from humans, they are also finding their way into our homes, e.g. as small cleaning helpers. Research on the other hand concentrates on larger, possibly humanoid robots, which may serve as mobility or household assistants, carry out exercises with patients in rehabilitation and act as production assistants in industry. As these applications involve the human and the robot sharing a workspace, the human has to be kept unharmed at all costs. Additionally, damage of the system and the environment should be avoided. This results in constraints on the joints, workspace, velocities, forces or torques of the system and requires a control scheme, which guarantees the adherence to the limits during task execution.

Possible solutions are found in constrained control and virtual wall rendering. The probably most widely used control schemes are the optimization-based model predictive control [1] and reference governor approach [2], which enforce input, output and state constraints. A large number of constraints or nonlinearities in the system dynamics may however prevent finding a solution in real-time. The invariance control approach [3] enforces output and state constraints by keeping the system controlled invariant within a constraint admissible subset of the state space, but implementation on real systems leads to chattering at the boundaries due to the digital implementation of the switching control law. Approaches creating virtual repulsive forces such as potential functions [4] and virtual fixtures [5], [6] may also be designed to enforce output and state constraints. However, both approaches neglect the inherent system dynamics in

All authors are with the Chair of Information-Oriented Control, Department of Electrical Engineering and Information Technology, Technical University of Munich, D-80290 München, Germany. manuel.rauscher@mytum.de, \{melanie.kimmel, hirche\}@etum.de determining a repulsive control action. As for higher-order dynamics, high accelerations or inertias, these dynamics are a substantial influence on the approach of a constraint, neither potential fields nor virtual fixtures are able to guarantee constraint adherence.

Although control barrier functions (CBFs) [7] are reminiscent of potential functions, CBF-based control design guarantees adherence to constraints. Knowledge about the system dynamics is combined with the CBFs in order to render a constraint admissible subset of the state space controlled invariant. In [8], this is achieved via model predictive control with CBFs. For improved real-time capabilities and to include performance specifications, Ames et al. [9] suggest a combination of CBFs with control Lyapunov functions (CLFs) via quadratic programming for cruise control. The approach is also successfully applied to bipedal robotic walking in [10] and for pendulum control [11]. The existing control strategies require, however, a joint control design for task execution and constraint enforcement. As there are several well-established control schemes to achieve a goal directed behavior for robotic systems, a combination of these methods with CBFs is desirable. Additionally, scenarios involving the interaction with humans require the enforcement of multiple and time-varying constraints, which has not been formally discussed for CBFs so far.

In this work, we introduce a novel CBF-based control structure, which allows the design of a constraint enforcing controller as an add-on to an already existing control loop. We show that by combining the existing control signal with CBFs via quadratic programming, the system adheres to an arbitrary number of possibly time-varying constraints, while following the original behavior whenever it is admissible. The capabilities of the control scheme are illustrated by experiments on a redundant anthropomorphic robotic manipulator, in which the CBF-based control law enforces multiple static and moving Cartesian workspace constraints in the presence of external forces, thus complementing the compliant behavior generated by an impedance controller.

The remainder of this paper is organized as follows: Section $\Pi$ introduces system requirements and the goals of the control design. In Section [III the theory of CBFs is revised. Section IV introduces the novel control scheme as an add-on to an existing control loop for multiple constraints. In Section $\nabla$ the experimental results are discussed. Section VI gives some concluding remarks.

Notation: Bold small and capital letters denote vectors and matrices, respectively. Dots are used to abbreviate time derivatives of low order $\dot{x}=\frac{\mathrm{d} x}{\mathrm{~d} t}$. The directional derivative of a scalar function $B(\boldsymbol{x})$ with respect to a vector field, i.e. 
the Lie derivative, is denoted by

$$
\mathcal{L}_{\boldsymbol{f}} B(\boldsymbol{x})=\frac{\partial B(\boldsymbol{x})}{\partial \boldsymbol{x}} \boldsymbol{f}(\boldsymbol{x})
$$

For a vector of vector fields $\boldsymbol{G}(\boldsymbol{x})=\left[\boldsymbol{g}_{1}(\boldsymbol{x}) \ldots \boldsymbol{g}_{m}(\boldsymbol{x})\right]$ the Lie derivative is defined as the vector of Lie derivatives in the directions $\boldsymbol{g}_{i}(\boldsymbol{x}): \mathcal{L}_{\boldsymbol{G}} B(\boldsymbol{x})=\left[\mathcal{L}_{\boldsymbol{g}_{\mathbf{1}}} B(\boldsymbol{x}) \ldots \mathcal{L}_{\boldsymbol{g}_{\boldsymbol{m}}} B(\boldsymbol{x})\right]$.

\section{Problem Statement}

We consider control affine, nonlinear systems of the form

$$
\left.\begin{array}{l}
\dot{\boldsymbol{x}}=\boldsymbol{f}(\boldsymbol{x})+\boldsymbol{G}(\boldsymbol{x}) \boldsymbol{u} \\
\boldsymbol{y}_{\text {out }}=\boldsymbol{h}_{\text {out }}(\boldsymbol{x})
\end{array}\right\}
$$

where $\boldsymbol{x} \in \mathbb{R}^{n}$ is the state with $\boldsymbol{x}(0)=\boldsymbol{x}_{0}$, the control input is $\boldsymbol{u} \in \mathcal{U} \subset \mathbb{R}^{m}$ with the set of admissible control values $\mathcal{U}$ and $\boldsymbol{f}: \mathbb{R}^{n} \rightarrow \mathbb{R}^{n}$ and $\boldsymbol{g}_{i}: \mathbb{R}^{n} \rightarrow \mathbb{R}^{n}$ are locally Lipschitz vector fields, where $\boldsymbol{G}=\left[\boldsymbol{g}_{1}, \ldots \boldsymbol{g}_{m}\right]$. The system output $\boldsymbol{y}_{\text {out }}(\boldsymbol{x})$ is given by a vector of output functions $\boldsymbol{h}_{\text {out }}: \mathbb{R}^{n} \rightarrow \mathbb{R}^{q}$. Note that a robotic system with the generalized coordinates $\boldsymbol{q}$, the mass matrix $\boldsymbol{M ( q )}$, the input torque $\tau$ and the Coriolis, centripetal and gravitational torques $\boldsymbol{C}(\boldsymbol{q}, \dot{\boldsymbol{q}}) \dot{\boldsymbol{q}}$ and $\boldsymbol{g}(\boldsymbol{q})$ has the generalized dynamics

$$
\boldsymbol{M}(\boldsymbol{q}) \ddot{\boldsymbol{q}}+\boldsymbol{C}(\boldsymbol{q}, \dot{\boldsymbol{q}}) \dot{\boldsymbol{q}}+\boldsymbol{g}(\boldsymbol{q})=\boldsymbol{\tau}
$$

thus being control affine. The goal is to design a control structure, which enforces the execution of a desired task while guaranteeing the adherence to known constraints. Arbitrary state and output constraints may only be enforced by a controllable system. This motivates the following assumption, which imposes only little restriction as robotic systems are controllable as long as singularities are avoided.

Assumption 1. The system (1) is controllable within the entire constrained space.

We aim for a control structure as depicted in Fig. 1. The system 11 is controlled by a combination of nominal

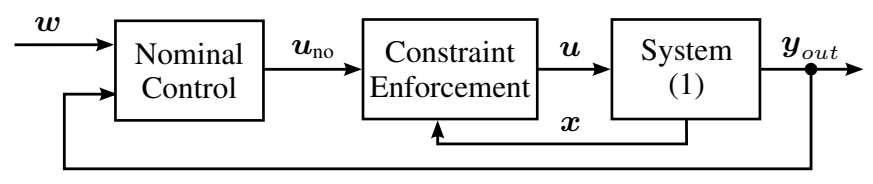

Fig. 1: Structure of the controlled system.

control and a constraint enforcing control. The advantage of the design as an add-on to nominal control is that constraints may be added to preexisting control loops and the nominal control law may be designed to achieve a desired performance and a Lyapunov stable tracking error

$$
\boldsymbol{e}=\boldsymbol{w}-\boldsymbol{y}_{\text {out }}
$$

without accounting for possible constraints. Constraint enforcing control monitors the constraint, the state $\boldsymbol{x}$ and the nominal control value $\boldsymbol{u}_{\text {no }}$. Ideally nominal control is applied whenever possible. In case of the danger of constraint violation the control input $\boldsymbol{u}$ is modified, such that the constraints hold. For the calculation of the modified control value so-called CBFs are used.

\section{CONTROL BASED ON CONTROL BARRIER FUNCTIONS}

In applications, requiring task execution while adhering to input, state or output constraints, control based on control barrier functions (CBFs) is promising. This section introduces CBFs for a single, static constraint [9], [10].

\section{A. Control Barrier Functions}

Essentially, a CBF $B(\boldsymbol{x})$ is a non-negative function with a small value for states far from a constraint and which grows to infinity as the state $\boldsymbol{x}$ approaches the constraint.

In order to find a suitable $\mathrm{CBF}$, the constraint on the system state $\boldsymbol{x}$ is encoded in a smooth constraint function $h(\boldsymbol{x})$. A value $h(\boldsymbol{x})>0$ indicates adherence, whereas $h(\boldsymbol{x}) \leq 0$ indicates a violation. This means that the constraint function has to be designed depending on the shape of the obstacle and measurement data such that $h(\boldsymbol{x})=0$ acts as a hull completely encasing the obstacle. The set of admissible states $\mathcal{C}$ is defined by

$$
\left.\begin{array}{l}
\mathcal{C}=\left\{\boldsymbol{x} \in \mathbb{R}^{n}: h(\boldsymbol{x})>0\right\} \\
\partial \mathcal{C}=\left\{\boldsymbol{x} \in \mathbb{R}^{n}: h(\boldsymbol{x})=0\right\}
\end{array}\right\}
$$

where $h: \mathbb{R}^{n} \rightarrow \mathbb{R}$ is a smooth, i.e. continuously differentiable, function and $\partial \mathcal{C}$ is the boundary of the set $\mathcal{C}$.

Definition 1. The system is constraint admissible, if its states stay within the admissible set of states for all times.

Naturally, the system may only be kept within the set of admissible states if such states exist. As $\mathcal{C}$ solely contains constraint admissible states $\boldsymbol{x}$, the goal is to design a control law which keeps the system within $\mathcal{C}$ for all future times, thus rendering the set controlled positive invariant.

Definition 2. Let $\boldsymbol{u} \in \mathcal{U}$ be a control value for the system (1). Let $\boldsymbol{x}_{0}=\boldsymbol{x}(0)$ be the initial state. A set $\mathcal{C}$ is called controlled positively invariant for the control value $\boldsymbol{u} \in \mathcal{U}$, if $\boldsymbol{x}_{0} \in \mathcal{C}$ implies that $\boldsymbol{x}(t) \in \mathcal{C}$ for all $t \geq 0$.

CBFs allow the implementation of such a control law. For a function $B(\boldsymbol{x})$ to qualify as a $\mathrm{CBF}$, it has to fulfill the following $\mathrm{CBF}$-properties $(C B F-p)$.

$\left(C B F-p_{1}\right)$ A valid $\mathrm{CBF}$ is a non-negative function on $\mathcal{C}$

$$
\inf _{\boldsymbol{x} \in \mathcal{C}} B(\boldsymbol{x}) \geq 0 .
$$

$\left(C B F-p_{2}\right)$ The barrier grows as a solution $\boldsymbol{x}$ approaches the constraint from inside the admissible set of states

$$
\lim _{\boldsymbol{x} \rightarrow \partial \mathcal{C}^{+}} B(\boldsymbol{x})=\infty .
$$

$\left(C B F-p_{3}\right)$ The CBF $B(\boldsymbol{x})$ grows with the growth rate

$$
\dot{B}(\boldsymbol{x}) \leq \frac{\gamma}{B(\boldsymbol{x})}, \text { where } \gamma>0 .
$$

Note that a CBF $B(\boldsymbol{x})$ behaves essentially like the inverse of a so-called class $\mathcal{K}$ function [12].

Definition 3. A continuous function $\alpha:[0, \infty) \rightarrow[0, \infty)$ belongs to class $\mathcal{K}$ if it is strictly increasing and $\alpha(0)=0$. 
A class $\mathcal{K}$ function $\alpha$ has the following properties [9], which are the desired properties for the $\mathrm{CBF} B(\boldsymbol{x})$ :

$$
\inf _{\boldsymbol{x} \in \mathcal{C}} \frac{1}{\alpha(h(\boldsymbol{x}))} \geq 0, \quad \lim _{\boldsymbol{x} \rightarrow \partial \mathcal{C}^{+}} \frac{1}{\alpha(h(\boldsymbol{x}))}=\infty .
$$

Hence, Def. 3 and $\left(C B F-p_{1}\right)$ to $\left(C B F-p_{3}\right)$ define CBFs.

Definition 4 (Control barrier function (CBF)). [9, Def. 2] Let a continuously differentiable $h: \mathbb{R}^{n} \rightarrow \mathbb{R}$ define $\mathcal{C} \subset \mathbb{R}^{n}$ as in (47). A locally Lipschitz function $B(\boldsymbol{x}): \mathcal{C} \rightarrow \mathbb{R}$ is a control barrier function (CBF), if its Lie derivatives $\mathcal{L}_{\boldsymbol{f}} B(\boldsymbol{x})$ and $\mathcal{L}_{G} B(\boldsymbol{x})$ are locally Lipschitz and if there exist class $\mathcal{K}$ functions $\alpha_{1}, \alpha_{2}$ and $\gamma>0$ such that for all $\boldsymbol{x} \in \mathcal{C}$

$$
\begin{gathered}
\frac{1}{\alpha_{1}(h(\boldsymbol{x}))} \leq B(\boldsymbol{x}) \leq \frac{1}{\alpha_{2}(h(\boldsymbol{x}))} \\
\inf _{\boldsymbol{u} \in \mathcal{U}}\left[\mathcal{L}_{\boldsymbol{f}} B(\boldsymbol{x})+\mathcal{L}_{\boldsymbol{G}} B(\boldsymbol{x}) \boldsymbol{u}-\frac{\gamma}{B(\boldsymbol{x})}\right] \leq 0 .
\end{gathered}
$$

The property $\left(\mathrm{CBF}-\mathrm{p}_{3}\right)$ provides the basis for the derivation of a control law. By enforcing this condition, constraint violation is prevented as it ensures that the growth of the function stops when the state approaches the constraint. A relationship between the CBFs and the control value $\boldsymbol{u}$ is derived by inserting (1) into $\left(C B F-p_{3}\right)$, which directly yields the set of admissible control values $\mathcal{K}_{B}(\boldsymbol{x})$.

$$
\mathcal{K}_{B}(\boldsymbol{x})=\left\{\boldsymbol{u} \in \mathcal{U}: \mathcal{L}_{\boldsymbol{f}} B(\boldsymbol{x})+\mathcal{L}_{\boldsymbol{G}} B(\boldsymbol{x}) \boldsymbol{u}-\frac{\gamma}{B(\boldsymbol{x})} \leq 0\right\}
$$

By applying $\boldsymbol{u} \in \mathcal{K}_{B}(\boldsymbol{x})$ to the system (1), the set $\mathcal{C}$ (4) is rendered controlled positively invariant [9, Corollary 1]. As the input only influences the system for $\mathcal{L}_{\boldsymbol{G}} B(\boldsymbol{x}) \neq \mathbf{0}, B(\boldsymbol{x})$ has to be designed such that $\dot{B}(\boldsymbol{x})$ depends directly on $\boldsymbol{u}$. For constraints of relative degree one, $\dot{h}(\boldsymbol{x})$ depends on $\boldsymbol{u}$ and $\mathcal{L}_{\boldsymbol{G}} B(\boldsymbol{x}) \neq \mathbf{0}$ holds for $B(\boldsymbol{x})=B(h(\boldsymbol{x}))$. However, often constraints on robotic systems, e.g. workspace or joint constraints, are of relative degree two, i.e. $\mathcal{L}_{\boldsymbol{G}} B(h(\boldsymbol{x}))=$ 0. Therefore, in the following section a CBF candidate for relative degree two constraint functions is introduced.

\section{B. CBFs for relative degree two constraints}

In torque-controlled robotic systems with the generalized dynamics (2), position constraints have relative degree two, i.e. $\ddot{h}(\boldsymbol{x})$ depends on the input. For such cases, the function

$$
B(\boldsymbol{x})=-\ln \left(\frac{h(\boldsymbol{x})}{1+h(\boldsymbol{x})}\right)+a_{E} \frac{b_{E} \dot{h}(\boldsymbol{x})^{2}}{1+b_{E} \dot{h}(\boldsymbol{x})^{2}}
$$

is introduced, where $h(\boldsymbol{x})$ and $\dot{h}(\boldsymbol{x})$ are the constraint function and its first time derivative. This is an admissible CBF, which is shown in [10, Theorem 2]. Note that choosing small design parameters $a_{E}, b_{E}>0$ causes the system to stop further from the constraints, whereas large values increase the risk of a violation in the presence of uncertainties, i.e. a reasonable trade-off has to be found depending on the application. The function includes $\dot{h}(\boldsymbol{x})$ in the CBF and therefore ensures $\mathcal{L}_{G} B(\boldsymbol{x}) \neq \mathbf{0}$. Note that based on the results from [10], the design of CBFs for constraints with arbitrary relative degree is also possible.
Both [9, Corollary 1] and [10, Theorem 2] provide the foundation for a control law which enforces a single, static constraint of relative degree one or two. Note that on approaching a constraint the value of a CBF goes to infinity due to $\left(C B F-p_{2}\right)$. This means, however, that CBFs only have a defined function value within the set of admissible states and therefore, they are not able to resolve constraint violations, e.g. due to inadmissible initial state values, an inaccurate system model or uncertainties in system and constraints. In the following section, we develop a control structure, which combines multiple constraints with an arbitrary desired nominal behavior.

\section{Multiple CBFs AND NOMinal CONTROL}

In this section, we develop a method, which enforces multiple constraints while executing a desired task. This requires the derivation of a condition on the control output, which will ensure the adherence to all constraints. We then show, that merging this condition with any stabilizing nominal control law via a quadratic program (QP) achieves the desired behavior.

\section{A. Multivariate Control Barrier Functions}

Let $l$ be the number of constraints on the system (1), which are expressed by continuously differentiable constraint functions $h_{i}: \mathbb{R}^{n} \rightarrow \mathbb{R}$, and which fulfill

$$
h_{i}(\boldsymbol{x})>0, \quad 1 \leq i \leq l
$$

for constraint admissible states. The set of the states $\boldsymbol{x}$ which fulfill the condition of each constraint $1 \leq i \leq l(9)$ is defined as the set of admissible states for multivariate constraints $\mathcal{H}$.

$$
\left.\begin{array}{rl}
\mathcal{H}=\left\{\boldsymbol{x} \in \mathbb{R}^{n}:\right. & \left.\forall 1 \leq i \leq l: \quad h_{i}(\boldsymbol{x})>0\right\} \\
\partial \mathcal{H}=\left\{\boldsymbol{x} \in \mathbb{R}^{n}:\right. & \exists 1 \leq j \leq l: \quad h_{j}(\boldsymbol{x})=0 \\
& \left.\wedge \forall 1 \leq i \leq l: \quad h_{i}(\boldsymbol{x}) \geq 0\right\}
\end{array}\right\}
$$

With definition (10) $\mathcal{H}$ is the intersection of the sets $\mathcal{C}_{i}$ (4) associated with the constraints $h_{i}(\boldsymbol{x})$. Therefore, admissible states only exist, if the individual constraints do not conflict.

Assumption 2. The set of admissible states $\mathcal{H}$ is not empty.

As the control goal is to keep the system within an admissible subset of the state space, which is only possible if such a subset exists, the assumption is straightforward. Note that in the case of a restricted workspace, the set of admissible states has to overlap with the workspace. Otherwise it is not possible to adhere to the constraints.

Let $B_{i}(\boldsymbol{x})$ be a CBF candidate corresponding to $h_{i}(\boldsymbol{x})$. Then $\mathcal{K}_{B_{i}}$ (7) is the set of admissible control values associated with $\mathcal{C}_{i}$ and the set of admissible control values for $\mathcal{H}$ is given by the intersection of all $\mathcal{K}_{B_{i}}$ for $1 \leq i \leq l$

$$
\begin{aligned}
\underline{\mathcal{K}}_{B}(\boldsymbol{x})=\{ & \boldsymbol{u} \in \mathcal{U}, \forall 1 \leq i \leq l: \\
& \left.\mathcal{L}_{\boldsymbol{f}} B_{i}(\boldsymbol{x})+\mathcal{L}_{G} B_{i}(\boldsymbol{x}) \boldsymbol{u}-\frac{\gamma_{i}}{B_{i}(\boldsymbol{x})} \leq 0\right\}
\end{aligned}
$$

where $\gamma_{i}>0$ are design parameters corresponding to each $B_{i}(\boldsymbol{x})$. With the properties of $\mathcal{H}$, the set of control values $\underline{\mathcal{K}}_{B}$ and [9, Corollary 1], invariance of $\mathcal{H}$ is shown. 
Corollary 1. Let $\mathcal{H} \subset \mathbb{R}^{n}$ be defined by (10). Let the functions $B_{i}(\boldsymbol{x})$ with $1 \leq i \leq l$ be the CBFs associated with the set $\mathcal{H}$. Let Assumptions 1$]$ and 2 hold. Then any Lipschitz continuous controller $\boldsymbol{u}(\boldsymbol{x}) \in \underline{\mathcal{K}}_{B}(\boldsymbol{x})$ applied to system (1) renders the set $\mathcal{H}$ positively invariant.

Proof. Each constraint $h_{i}$ with $1 \leq i \leq l$ is associated with a $\mathrm{CBF} B_{i}$, the set of admissible states $\mathcal{C}_{i}$ and the set of admissible control values $\mathcal{K}_{B_{i}}$. By Assumption 2 the constraints do not conflict. By Assumption 1 all state variables of the system 1 are controllable. Therefore $\underline{\mathcal{K}}_{B}(\boldsymbol{x})$ is not empty and there exists a $\boldsymbol{u} \in \underline{\mathcal{K}}_{B}(\boldsymbol{x})$.

The set $\underline{\mathcal{K}}_{\boldsymbol{B}}(\boldsymbol{x})$ is the intersection of the individual $\mathcal{K}_{B_{i}}(\boldsymbol{x})$ with $1 \leq i \leq l$. Hence if $\boldsymbol{u}$ lies within $\underline{\mathcal{K}}_{B}(\boldsymbol{x})$, it lies also within all $\mathcal{K}_{B_{i}}(\boldsymbol{x})$. Therefore, by [9, Corollary 1], $\boldsymbol{u}$ renders all $\mathcal{C}_{i}, 1 \leq i \leq l$, invariant. As $\mathcal{H}$ is the intersection of all $\mathcal{C}_{i}, \mathcal{H}$ is rendered invariant by $\boldsymbol{u} \in \underline{\mathcal{K}}_{B}(\boldsymbol{x})$.

Intuitively, Corollary 1 shows that by choosing only control values from the set $\underline{\mathcal{K}}_{B}(\boldsymbol{x})$, the system is forced to stay within the admissible set of states $\mathcal{H}$ for all times, thus avoiding a violation of the constraints. Additionally, a Lipschitz continuous $\boldsymbol{u}(\boldsymbol{x})$ avoids large, instantaneous changes in the control value, thus enabling a torque-controlled robotic system to follow the desired torque and reducing the overall stress of the system. Based on these results, nominal control is unified with the CBF approach for multiple constraints.

\section{B. Combining the Control Goal and the Constraints}

In this section, we show that it is possible to combine CBFs with arbitrary nominal control laws using a quadratic program (QP). The aim of the combined control law is to apply nominal control whenever possible, which is formulated as an optimization problem, minimizing the error $\boldsymbol{e}_{u}$

$$
\boldsymbol{e}_{u}=\boldsymbol{u}_{\mathrm{no}}-\boldsymbol{u}
$$

between the nominal control signal $\boldsymbol{u}_{\text {no }}$ and the applied control value $\boldsymbol{u}$. The squared norm of the error

$$
\left\|\boldsymbol{e}_{u}\right\|^{2}=\boldsymbol{u}^{\top} \boldsymbol{u}-2 \boldsymbol{u}_{\mathrm{no}}^{\top} \boldsymbol{u}+\boldsymbol{u}_{\mathrm{no}}^{\top} \boldsymbol{u}_{\mathrm{no}}
$$

is used as objective function. Neglecting the last term of (13), as it is constant in a minimization with respect to $\boldsymbol{u}$, yields the optimization problem to obtain a desired control action $\boldsymbol{u}^{*} \in \mathbb{R}^{m}$

$$
\begin{aligned}
\boldsymbol{u}^{*}(\boldsymbol{x})=\underset{\boldsymbol{u} \in \mathbb{R}^{m}}{\operatorname{argmin}} \boldsymbol{u}^{\top} \boldsymbol{u}-2 \boldsymbol{u}_{\mathrm{no}}^{\top} \boldsymbol{u} \\
\text { s.t. } \quad \boldsymbol{A}(\boldsymbol{x}) \boldsymbol{u} \leq \boldsymbol{b}(\boldsymbol{x})
\end{aligned}
$$

where $\boldsymbol{u}_{\text {no }} \in \mathcal{U}_{\text {no }} \subset \mathbb{R}^{m}$ is a locally Lipschitz continuous nominal control signal and the constraints in terms of CBFs with $\boldsymbol{A}(\boldsymbol{x}) \in \mathbb{R}^{l \times m}$ and $\boldsymbol{b}(\boldsymbol{x}) \in \mathbb{R}^{l}$

$$
\begin{gathered}
\boldsymbol{A}(\boldsymbol{x})=\left[\mathcal{L}_{\boldsymbol{G}} B_{i}\right]=\left[\begin{array}{ccc}
\mathcal{L}_{\boldsymbol{g}_{1}} B_{1} & \cdots & \mathcal{L}_{\boldsymbol{g}_{\boldsymbol{m}}} B_{1} \\
\vdots & & \vdots \\
\mathcal{L}_{\boldsymbol{g}_{1}} B_{l} & \cdots & \mathcal{L}_{\boldsymbol{g}_{m}} B_{l}
\end{array}\right], \\
\boldsymbol{b}(\boldsymbol{x})=\left[\frac{\gamma_{i}}{B_{i}}-\mathcal{L}_{\boldsymbol{f}} B_{i}\right]=\left[\begin{array}{c}
\frac{\gamma_{1}}{B_{1}}-\mathcal{L}_{\boldsymbol{f}} B_{1} \\
\vdots \\
\frac{\gamma_{l}}{B_{l}}-\mathcal{L}_{\boldsymbol{f}} B_{l}
\end{array}\right]
\end{gathered}
$$

are included as inequality constraints imposed upon $\boldsymbol{u}$, such that $\boldsymbol{u}^{*} \in \underline{\mathcal{K}}_{\boldsymbol{B}}(\boldsymbol{x})$ (11) holds. Note that input constraints may be included straightforwardly by appending the corresponding entries to $\boldsymbol{A}(\boldsymbol{x})$ and $\boldsymbol{b}(\boldsymbol{x})$. However, the input and state constraints have to be designed carefully as the control scheme does not provide solutions for contradicting constraints or loss of manipulability, which renders the optimization infeasible.

Theorem 1. Let a control affine system (1) with $l$ state and output constraints and a locally Lipschitz nominal control signal $\boldsymbol{u}_{n o}$ be given. Let $B_{i}, 1 \leq i \leq l$, be control barrier functions associated with the constraints and let $\mathcal{H}$ as in (10) be the corresponding set of admissible states. Let Assumptions 1 and 2 hold. Then, the control law $\boldsymbol{u}^{*}(\boldsymbol{x})$ obtained by solving the quadratic program 14 is Lipschitz continuous and renders the set $\mathcal{H}$ positively invariant.

\section{Proof of Theorem 1 .}

The proof is conducted in two steps. First, uniqueness and Lipschitz continuity of the solution is proved. In a second step the invariance of the set $\mathcal{H}$ is shown.

Step 1: Uniqueness and Lipschitz continuity:

Let $\boldsymbol{u}^{*}(\boldsymbol{x})$ be a solution of (14) at a point $\boldsymbol{x} \in \mathbb{R}^{n}$. According to [13, Theorem 1], if the conditions

(i) $w^{*}(\boldsymbol{x})>0$, where $w^{*}(\boldsymbol{x})$ is the solution of the linear program

$$
\begin{aligned}
& w^{*}(\boldsymbol{x})=\max _{(\boldsymbol{u}, w) \in \mathbb{R}^{m+1}} w \\
& \text { s.t. }\left[\begin{array}{ll}
\boldsymbol{A}(\boldsymbol{x}) & \mathbf{1}_{l \times 1}
\end{array}\right]\left[\begin{array}{c}
\boldsymbol{u} \\
w
\end{array}\right] \leq \boldsymbol{b}(\boldsymbol{x}),
\end{aligned}
$$

(ii) $\boldsymbol{A}(\boldsymbol{x})$ and $\boldsymbol{b}(\boldsymbol{x})$ are Lipschitz continuous at $\boldsymbol{x}$,

(iii) $\boldsymbol{u}_{\mathrm{no}}(\boldsymbol{x})$ is Lipschitz continuous at $\boldsymbol{x}$,

with $\boldsymbol{A}(\boldsymbol{x}) \in \mathbb{R}^{l \times m}$ and $\boldsymbol{b}(\boldsymbol{x}) \in \mathbb{R}^{l}$ from (15) hold at a state $\boldsymbol{x} \in \mathbb{R}^{n}$, then the solution $\boldsymbol{u}^{*}(\boldsymbol{x})$ of the QP (14) is unique and Lipschitz continuous at $\boldsymbol{x}$.

Condition ( $i$ ) is based on the Mangasarian-Fromovitz constraint qualification [14]. If the constraint qualification holds, which is expressed by $w^{*}(\boldsymbol{x})>0$, the solution of the QP (14) is unique at $\boldsymbol{x}$. Conditions (ii) and (iii) are necessary for the Lipschitz continuity of the solution of (14). To verify Condition (i), the linear program (LP) 16 is rewritten to include the constraints (17) in the objective function

$$
w^{*}(\boldsymbol{x})=\max _{\substack{\boldsymbol{u} \in \mathbb{R}^{m} \\ 1 \leq i \leq l}} \frac{\gamma}{B_{i}(\boldsymbol{x})}-\mathcal{L}_{\boldsymbol{f}} B_{i}(\boldsymbol{x})-\mathcal{L}_{\boldsymbol{G}} B_{i}(\boldsymbol{x}) \boldsymbol{u} .
$$

This is possible since the set $\mathcal{H}$ is not empty by Assumption 2, which means that the constraints do not conflict and the optimization is strictly convex. Substituting

$$
\dot{B}(\boldsymbol{x})=\frac{\partial B(\boldsymbol{x})}{\partial \boldsymbol{x}} \underbrace{(\boldsymbol{f}(\boldsymbol{x})+\boldsymbol{G}(\boldsymbol{x}) \boldsymbol{u})}_{\dot{\boldsymbol{x}}}=\mathcal{L}_{\boldsymbol{f}} B(\boldsymbol{x})+\mathcal{L}_{\boldsymbol{G}} B(\boldsymbol{x}) \boldsymbol{u}
$$

yields

$$
w^{*}(\boldsymbol{x})=\max _{\substack{\boldsymbol{u} \in \mathbb{R}^{m} \\ 1 \leq i \leq l}} w(\boldsymbol{x})
$$


with $w(\boldsymbol{x})=\frac{\gamma}{B_{i}(\boldsymbol{x})}-\dot{B}_{i}(\boldsymbol{x})$. As the CBFs fulfill $\left(C B F-p_{3}\right), w(x) \geq 0$ holds. By definition (10), the boundary $\partial \mathcal{H}$ is not part of $\mathcal{H}$, which means that $w(\boldsymbol{x})>0$ holds within $\mathcal{H}$, as $w(\boldsymbol{x})=0$ holds only on $\partial \mathcal{H}$. As a result, $w^{*}(\boldsymbol{x})>0$ is fulfilled on $\mathcal{H}$, confirming Condition (i). Condition (ii) holds for all $\boldsymbol{x} \in \mathcal{H}$ as local Lipschitz continuity of $\boldsymbol{A}(\boldsymbol{x})$ and $\boldsymbol{b}(\boldsymbol{x})$ follows from Definition 4, which demands that a CBF $B_{i}$ and its Lie derivatives $\mathcal{L}_{\boldsymbol{f}} B_{i}(\boldsymbol{x})$ and $\mathcal{L}_{G} B_{i}(\boldsymbol{x})$ are locally Lipschitz. As the nominal control signal $\boldsymbol{u}_{\text {no }}$ is assumed to be locally Lipschitz, Condition (iii) holds as well. Therefore, all three conditions hold for all $\boldsymbol{x} \in \mathcal{H}$ and the control value $\boldsymbol{u}^{*}(\boldsymbol{x})$ obtained by solving the QP (14) is unique and Lipschitz for all $\boldsymbol{x} \in \mathcal{H}$.

Step 2: Invariance of $\mathcal{H}$ :

The constraint of (14) ensures that $\boldsymbol{u}^{*}(\boldsymbol{x})$ lies in $\underline{\mathcal{K}}_{B}$. Step 1 shows that the solution $\boldsymbol{u}^{*}(\boldsymbol{x})$ is Lipschitz continuous. With Assumptions 1 and 2 Corollary 11 is applicable and $\boldsymbol{u}^{*}(\boldsymbol{x})$ renders the set $\mathcal{H}$ positively invariant.

Theorem 1 shows, that the CBF approach is applicable to nominally controlled systems with multiple, not conflicting constraints. By solving the QP (14) the invariance of $\mathcal{H}(10)$ and a minimal error $\boldsymbol{e}_{u}$ are obtained simultaneously. Thus, nominal control is followed whenever the state $\boldsymbol{x}$ is sufficiently far away from the bounds. Additional characteristics and capabilities of the approach are stated without proof in the following remarks.

Remark 1: Theorem 11 does not require nominal control to be Lyapunov stable to render $\mathcal{H}$ controlled invariant. In order to achieve a desired control or performance goal, however, stability is necessary for reaching that goal.

Remark 2: The approach is also applicable with multiple constraints of arbitrary relative degree $r>2$ as Theorem 1 holds independently from the relative degree. However, as the CBF has to fulfill Definition 4 and $\mathcal{L}_{G} B(\boldsymbol{x}) \neq \mathbf{0}$, the design of the CBF may become more complicated.

Remark 3: Enforcing a constraint with relative degree $r$ on a system with more than $r$ states results in uncontrolled internal dynamics, which may cause undesired behavior. For robotic systems of the form (2), this is the case for upper bounds on the torques, accelerations and velocities. This results in a deviation from the desired trajectory and a slower motion than expected from nominal control. It does, however, not cause unexpectedly fast or large motions and results in the desired constraint admissible behavior.

Remark 4: The approach and Theorem 1 are also applicable with non-conflicting, time-dependent constraints $h_{i}(\boldsymbol{x}, t)$. If the CBF properties are fulfilled for all instants of time $t$ and the $h_{i}(\boldsymbol{x}, t)$ are sufficiently smooth, the only difference is an additional partial time derivative in $\dot{B}_{i}=\frac{\partial B_{i}}{\partial x} \dot{\boldsymbol{x}}+\frac{\partial B_{i}}{\partial t}$, which changes the conditions in the sets of admissible control values for single $\mathcal{K}_{B}(\boldsymbol{x})$ and multiple constraints $\underline{\mathcal{K}}_{B}(\boldsymbol{x})$ to

$$
\mathcal{L}_{\boldsymbol{f}} B_{i}(\boldsymbol{x})+\mathcal{L}_{\boldsymbol{G}} B_{i}(\boldsymbol{x}) \boldsymbol{u}+\frac{\partial B_{i}}{\partial t}-\frac{\gamma_{i}}{B_{i}(\boldsymbol{x})} \leq 0 .
$$

\section{EXPERIMENTAL EVALUATION}

In this section, the developed control scheme is verified in an experiment on an anthropomorphic manipulator with seven degrees of freedom [15]. A desired compliant behavior is achieved by implementing an impedance control law, representing nominal control. Naturally, other nominal control laws may be applied and yield a different behavior. By using impedance control, however, the experiment illustrates that even if the nominal control input is influenced by a human-generated signal, the proposed control scheme achieves compliance with the constraints. Simultaneously, multiple Cartesian workspace constraints are enforced using CBFs and a QP.

Note that although in the following constraints are imposed solely on the Cartesian position of the end effector, in general it is also possible to introduce constraints on joint level, on the entire manipulator or on the respective velocities by defining appropriate constraint functions and CBFs.

\section{A. Experimental Procedure}

In a first experiment, the Cartesian end effector position $\boldsymbol{p}=\left[\begin{array}{lll}p_{1} & p_{2} & p_{3}\end{array}\right]^{\top}$ is constrained in the three orthogonal directions as depicted in Fig. 2 The constraint functions

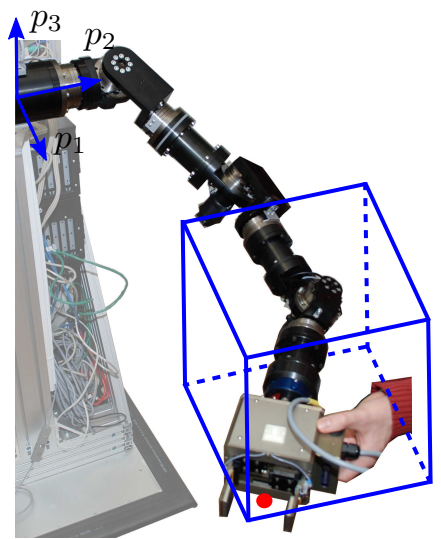

Fig. 2: Manipulator with Cartesian box constraints: • tool center point and - constraints.

$$
\begin{aligned}
h_{\text {up }, i}(\boldsymbol{p}) & =p_{i, \text { max }}-p_{i, \mathrm{~d}} \\
h_{\text {low }, i}(\boldsymbol{p}) & =p_{i, \mathrm{~d}}-p_{i, \text { min }}
\end{aligned}
$$

where $i \in\{1,2,3\}$ and $p_{i, \min }$ and $p_{i, \max }$ are the lower and upper bounds, respectively.

In a second experiment, a single, moving, spherical constraint is enforced. While the radius $r_{s}$ of the constraint is constant, the time-dependent Cartesian center position $\boldsymbol{c}(t)$ of the obstacle is detected by the Qualisys Motion Tracking System. In this case, the constraint function is determined by

$$
h(\boldsymbol{p}, t)=\|\boldsymbol{c}(t)-\boldsymbol{p}\|_{2}-r_{s},
$$

where $\|\cdot\|_{2}$ denotes the Euclidean vector norm.

The control structure of both experiments is depicted in Fig. 3 The control law (14) with CBFs of the form (8) is combined with nominal control and a simplified model of the robot dynamics to generate a constraint admissible reference trajectory $\boldsymbol{p}_{\mathrm{d}}$ as input to the position-controlled robotic system. An impedance control law [16], which enables the system to react to the external force $f_{\text {ext }} \in \mathbb{R}^{m}$, measured 


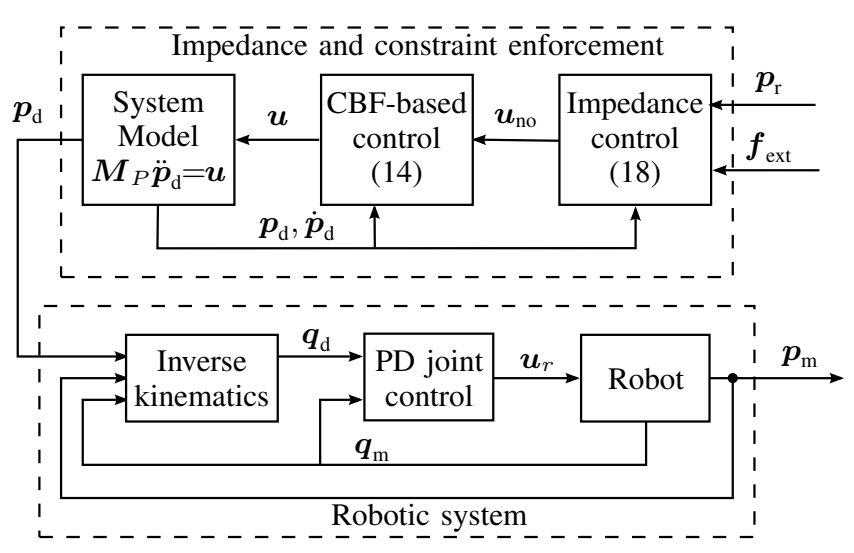

Fig. 3: Control structure of the experiments.

by a JR3 sensor attached to the end effector, provides the nominal control signal $\boldsymbol{u}_{\text {no }}$.

$$
\boldsymbol{u}_{\mathrm{no}}=\boldsymbol{f}_{\mathrm{ext}}+\boldsymbol{M}_{P} \ddot{\boldsymbol{p}}_{\mathrm{r}}+\boldsymbol{D}_{P}\left(\dot{\boldsymbol{p}}_{\mathrm{r}}-\dot{\boldsymbol{p}}_{\mathrm{d}}\right)+\boldsymbol{K}_{P}\left(\boldsymbol{p}_{\mathrm{r}}-\boldsymbol{p}_{\mathrm{d}}\right)
$$

The control structure is implemented using the Matlab/Simulink Real Time Workshop. The code runs with a sampling rate of $1 \mathrm{kHz}$. The real time QP solver is generated by CVXGEN [17]. The necessary parameters are given in Tab. I. Note that the parameters $a_{E}$ and $b_{E}$ may differ for each constraint, i.e. in a setup with multiple constraints, each constraint may be assigned a different set of parameters.

\begin{tabular}{|c|c|c|}
\hline & Parameter & Value \\
\hline $\begin{array}{l}\text { Sampling time } \\
\text { Mass of robot }\end{array}$ & $\begin{array}{l}T_{A} \\
M_{P} \\
\end{array}$ & 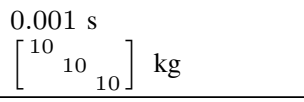 \\
\hline $\begin{array}{l}\text { Impedance } \\
\text { control }\end{array}$ & $\begin{array}{l}\boldsymbol{D}_{P} \\
\boldsymbol{K}_{P} \\
\end{array}$ & $\begin{array}{l}80 \cdot \boldsymbol{I}_{3} \mathrm{Ns} / \mathrm{m} \\
200 \cdot \boldsymbol{I}_{3} \mathrm{~N} / \mathrm{m}\end{array}$ \\
\hline $\begin{array}{l}\text { CBF-based } \\
\text { control } \\
\text { (static) }\end{array}$ & $\begin{array}{l}\gamma \\
a_{E} \\
b_{E}\end{array}$ & $\begin{array}{l}10 \\
100 \\
10 \\
\end{array}$ \\
\hline $\begin{array}{l}\text { CBF-based } \\
\text { control } \\
\text { (sphere) }\end{array}$ & $\begin{array}{l}\gamma \\
a_{E} \\
b_{E}\end{array}$ & $\begin{array}{l}10 \\
1 \\
1\end{array}$ \\
\hline $\begin{array}{l}\text { Constraint } \\
\text { parameters }\end{array}$ & $\begin{array}{l}\boldsymbol{p}_{\max } \\
\boldsymbol{p}_{\min } \\
r_{s}\end{array}$ & 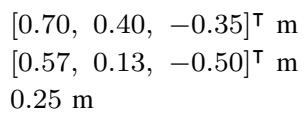 \\
\hline
\end{tabular}

TABLE I: Model and Control parameters.

During the experiments, the nominal control input forces the end effector to follow a given, desired trajectory and to react compliantly to a human input. Nominal control does, however, not take any constraints into account, i.e. the generated nominal control input may induce a violation of constraints. Therefore, the developed control scheme based on CBFs replaces the nominal control input by a constraint enforcing input.

\section{B. Experimental Results}

In Fig. 4, the Cartesian position of the end effector and the measured external forces during the first experiment with six static constraints are depicted. During the first few seconds,
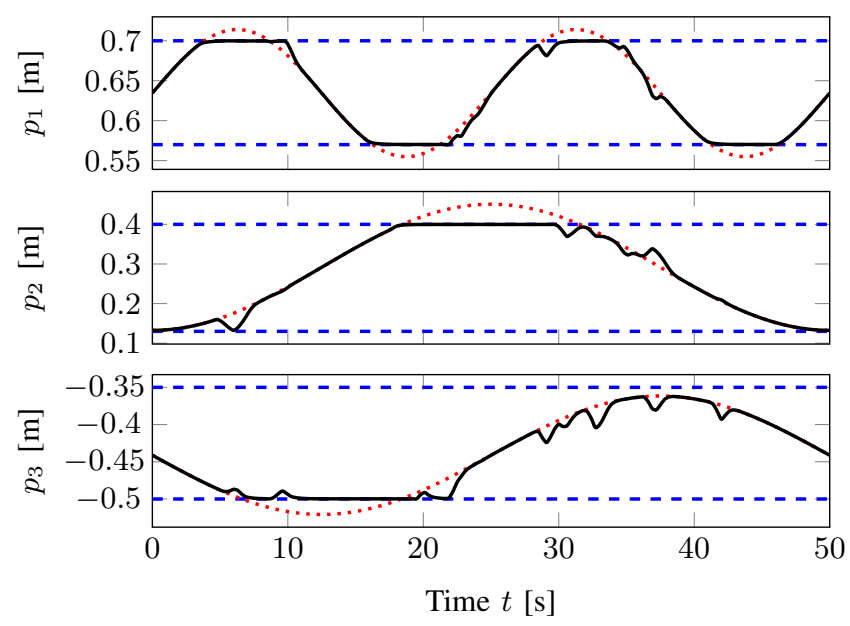

(a) End effector position: ...... reference trajectory $\boldsymbol{p}_{\mathrm{r}}$, --bounds and $\longrightarrow$ desired position of the end effector $\boldsymbol{p}_{\mathrm{d}}$.

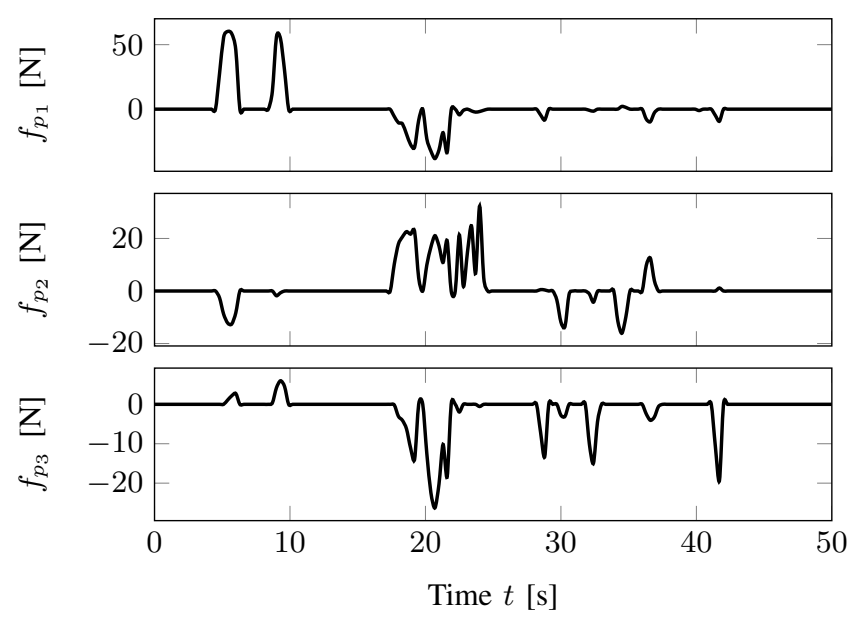

(b) Measured external forces: $\boldsymbol{f}_{\mathrm{ext}}=\left[f_{p_{1}} f_{p_{2}} f_{p_{3}}\right]^{\top}$.

Fig. 4: Experiment with six static Cartesian constraints.

the desired position follows the reference trajectory, as the end effector is away from the bounds and no forces are applied. If the end effector is at a distance from the constraint and an external force is applied, cf. $p_{3}$ from $t=25 \mathrm{~s}$ to $t=45 \mathrm{~s}$, or if the system is close to a constraint and the force is directed away from the limit, cf. $p_{3}$ from $t=5 \mathrm{~s}$ to $t=10 \mathrm{~s}$, the end effector gives way to the external force. This shows the effect of the nominal impedance control law.

Figure 4a also shows that the desired trajectory never violates a constraint even though the reference trajectory does not adhere to the constraints, cf. $p_{1}$ from $t=3 \mathrm{~s}$ to $t=9 \mathrm{~s}$. In addition, when the trajectory is close to a boundary and forces are applied pushing the end effector towards the limit, cf. $p_{1}$ and $p_{2}$ from $t=15 \mathrm{~s}$ to $t=25 \mathrm{~s}$, no violation occurs. Hence the controller enforces the constraints in the 
presence of external forces and even if the reference leaves the admissible set of states.

The measured position of the end effector $\boldsymbol{p}_{\mathrm{m}}$ is not included in the plots, as it coincides almost exactly with the desired position and gives no additional value. However, the measured position shows some negligible violations $(<$ $1 \mathrm{~mm}$ ) of the constraint, which are due to measurement noise and an inexact system modeling in the trajectory generation, where Coriolis, centripetal and gravity effects are neglected. These slight violations do, however, not challenge the general feasibility of the approach.

Figure 5 shows the results of the second experiment, where the obstacle moves, the reference position is constant and no forces are applied. During the first few seconds, the

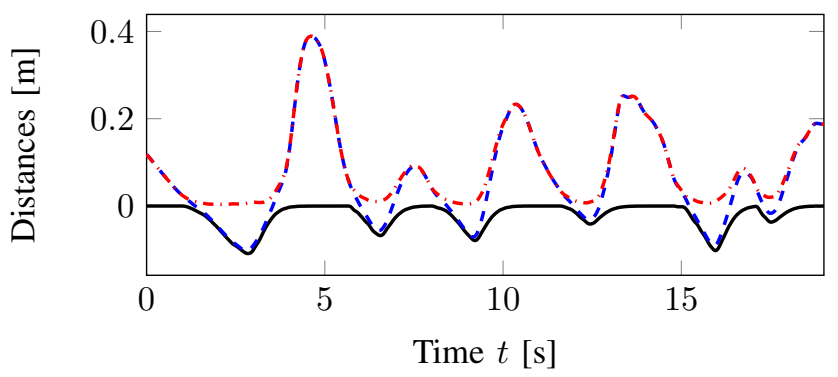

Fig. 5: Reaction of the end effector to the moving constraint: - the negative deviation of the end effector from the reference position $-\left\|\boldsymbol{p}_{\mathrm{m}}-\boldsymbol{p}_{\mathrm{r}}\right\|_{2},-\cdots$ the distance between the surface of the sphere and the reference position $\| c-$ $\boldsymbol{p}_{\mathrm{r}} \|_{2}-r_{s}$ and $\cdots \cdots$ the constraint function $h(\boldsymbol{p}, t)$.

constraint is at a distance from the end effector and the end effector holds the desired position, which leads to no deviation as depicted in the figure. However, if the constraint moves towards the end effector, i.e. $\left\|\boldsymbol{c}-\boldsymbol{p}_{\mathrm{r}}\right\|_{2}-r_{s}$ decreases in value, a deviation from the reference position occurs as the system has to move to avoid a violation of the constraint. The almost coinciding blue, dashed and black lines show that the end effector only deviates as much from the desired position as is necessary to avoid a violation. This is also emphasized by the constraint function, which illustrates the distance between the end effector and the constraint and which approaches zero in these cases.

Both experiments illustrate the capabilities of the developed control scheme. It combines the desired nominal behavior with the given limits to achieve a constraint admissible behavior and it is able to handle time-dependent constraints.

\section{CONCLUSiON}

In this paper, a novel design of constraint enforcing CBFbased control as an add-on to an existing control loop is introduced. The approach is applicable to nonlinear control affine systems with an existing nominal control structure. It enforces an arbitrary number of constraints, while allowing for nominal system behavior as long as it is constraint admissible. In two experiments on a redundant anthropomorphic manipulator, the control scheme combines compliant behavior, generated by a nominal impedance control scheme, with static Cartesian workspace constraints and a moving spherical constraint. Future work may focus on the issue that CBF-based control is currently not able to resolve constraint violations.

\section{ACKNOWLEDGEMENTS}

The research leading to these results has received funding from the European Union Seventh Framework Programme FP7/2007-2013 within the ERC Starting Grant Control based on Human Models (con-humo) under the grant agreement no. 337654 .

\section{REFERENCES}

[1] D. Mayne, J. Rawlings, C. Rao, and P. Scokaert, "Constrained model predictive control: Stability and optimality," Automatica, vol. 36, no. 6, pp. 789-814, 2000.

[2] E. Gilbert and I. Kolmanovsky, "Nonlinear tracking control in the presence of state and control constraints: a generalized reference governor," Automatica, vol. 38, no. 12, pp. 2063-2073, 2002.

[3] J. Wolff and M. Buss, "Invariance Control Design for Nonlinear Control Affine Systems under Hard State Constraints," in Proc. of the NOLCOS'2004 Symposium on Nonlinear Control Systems, Stuttgart, Germany, Sept. 2004, pp. 711-716.

[4] E. Rimon and D. Koditschek, "Exact Robot Navigation Using Artificial Potential Functions," IEEE Transactions on Robotics and Automation, vol. 8, no. 5, pp. 501-518, 1992.

[5] L. Rosenberg, "Virtual Fixtures: Perceptual Tools for Telerobotic Manipulation," in Proc. of the IEEE Virtual Reality Annual International Symposium, 1993, pp. 76-82.

[6] J. J. Abbott, P. Marayong, and A. M. Okamura, "Haptic Virtual Fixtures for Robot-Assisted Manipulation," in Robotics Research, R. B. S. Thrun and H. D. Whyte, Eds. Springer STAR series, 2007, pp. 49-64.

[7] P. Wieland and F. Allgöwer, "Constructive safety using control barrier functions," in Proc. of the 7th IFAC Symposium on Nonlinear Control Systems, vol. 7, no. 1, 2007, pp. 462-467.

[8] A. Wills and W. Heath, "Barrier function based model predictive control," Automatica, vol. 40, no. 8, pp. 1415-1422, 2004.

[9] A. Ames, J. Grizzle, and P. Tabuada, "Control Barrier Function based Quadratic Programs with Application to Adaptive Cruise Control," in Proc. of the IEEE 53rd Annual Conference on Decision and Control (CDC), 2014, pp. 6271-6278.

[10] S. Hsu, X. Xu, and A. Ames, "Control Barrier Function based Quadratic Programs with Application to Bipedal Robotic Walking," in Proc. of the American Control Conference (ACC), 2015, pp. 45424548.

[11] G. Wu and K. Sreenath, "Safety-Critical and Constrained Geometric Control Synthesis using Control Lyapunov and Control Barrier Functions for Systems Evolving on Manifolds," in Proc. of the American Control Conference (ACC), 2015, pp. 2038-2044.

[12] H. Khalil, Nonlinear Systems, 3rd ed. Prentice Hall, 1996.

[13] B. Morris, M. Powell, and A. Ames, "Sufficient Conditions for the Lipschitz Continuity of QP-based Multi-Objective Control of Humanoid Robots," in Proc. of the IEEE 52nd Annual Conference on Decision and Control (CDC), 2013, pp. 2920-2926.

[14] O. Mangasarian and S. Fromovitz, "The Fritz John Necessary Optimaityl Conditions in the Presence of Equality and Inequality Constraints," Journal of Mathematical Analysis and Applications, vol. 17, pp. 37-47, 1967.

[15] B. Stanczyk, "Development and Control of an Anthropomorphic Telerobotic System," Ph.D. dissertation, Technische Universität München, 2006.

[16] A. Albu-Schäffer and G. Hirzinger, "Cartesian Impedance Control Techniques for Torque Controlled Light-Weight Robots," in Proc. of the IEEE International Conference on Robotics and Automation, vol. 1, 2002, pp. 657-663.

[17] J. Mattingley and S. Boyd, "CVXGEN: a code generator for embedded convex optimization," Optimization and Engineering, vol. 13, no. 1, pp. 1-27, 2011. 